\title{
Generative Models for Extrapolation Prediction in
}

\section{Materials Informatics}

Kan Hatakeyama-Sato* and Kenichi Oyaizu*

Department of Applied Chemistry Waseda University, Tokyo 169-8555, Japan

*oyaizu@waseda.jp 

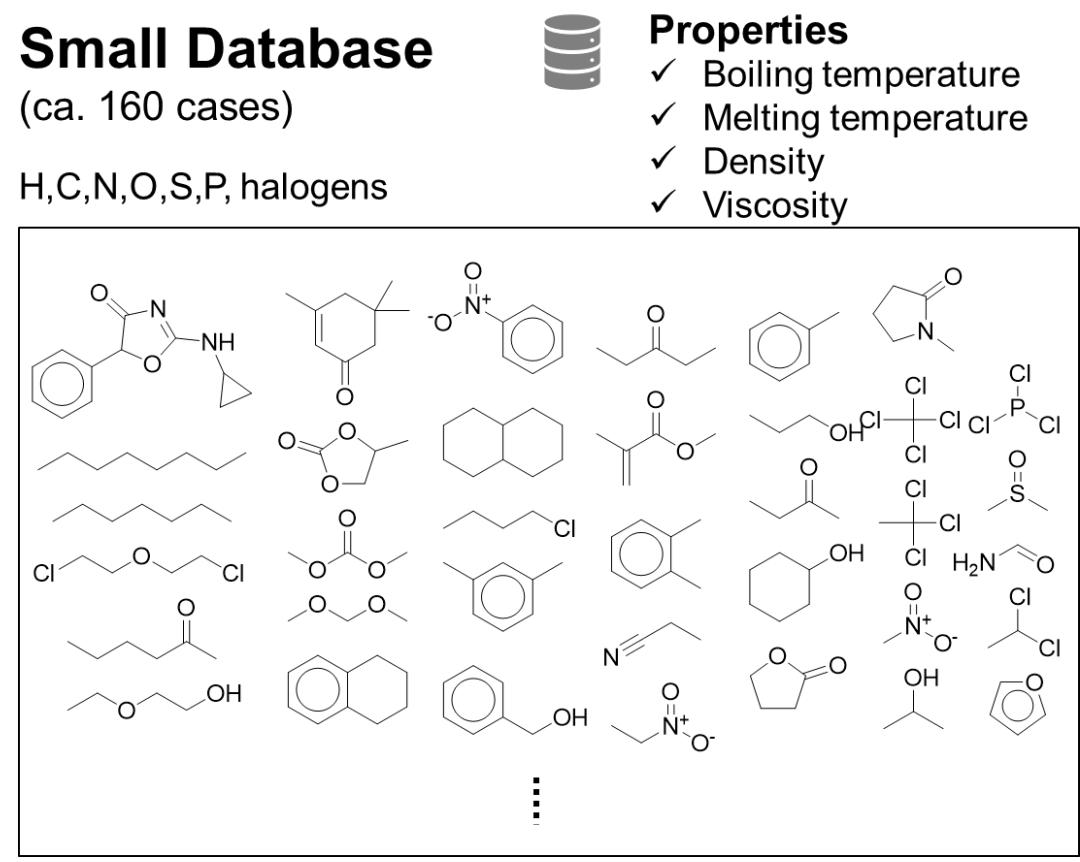

a)

Integrated Database (ca. 12000 cases)

\section{Properties}

More than 25 types of physical and chemical properties

$\mathrm{H}, \mathrm{B}, \mathrm{C}, \mathrm{N}, \mathrm{O}, \mathrm{S}, \mathrm{P}$, halogens

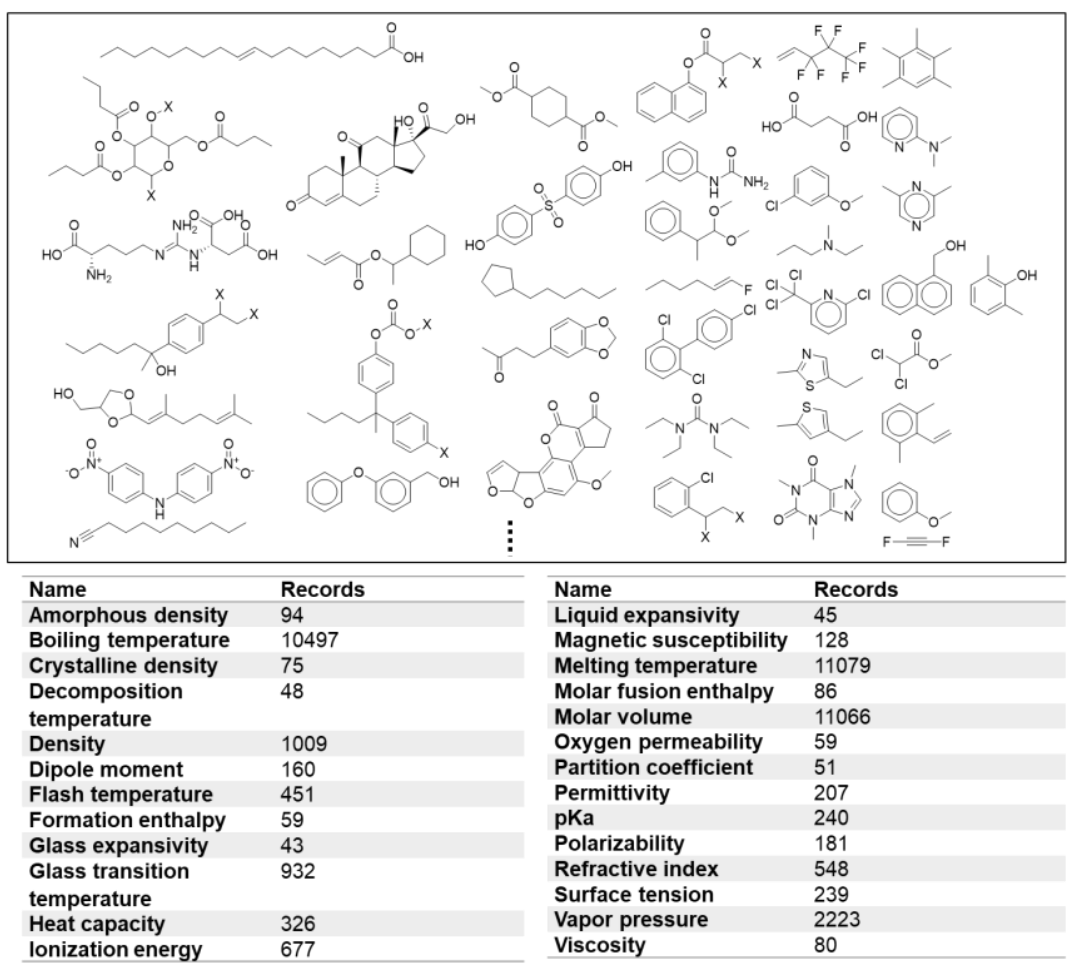

b)

Figure S1. Information of chemical compound database used in this study. a) Small and b) integrated databases contain experimental properties of versatile molecules. 


\begin{tabular}{|c|c|c|c|}
\hline Descriptor & $\begin{array}{l}\text { Dime } \\
\text { nsion }\end{array}$ & Type & \\
\hline FP(Avalon) & 512 & Binary & Avalon fingerprint. \\
\hline $\operatorname{Desc}(2 \mathrm{D})$ & 200 & Continuous & $\begin{array}{l}\text { Basic molecular descriptors generated by RDKit. a) } \\
\text { Ignores 3D structures. }\end{array}$ \\
\hline $\operatorname{Desc}(3 \mathrm{D})$ & 629 & Continuous & $\begin{array}{l}\text { Descriptors related to 3D molecular strucrures, } \\
\text { generated by DRKit. }{ }^{\text {) }}\end{array}$ \\
\hline HSPiP & 56 & Continuous & $\begin{array}{l}\text { Empirical molecular properties and descriptors } \\
\text { calculated by HSPiP package. }\end{array}$ \\
\hline PM7 & 13 & Continuous & Molecular properties calculated by PM7 method. \\
\hline Neural $(b p)^{c)}$ & 32 & Continuous & $\begin{array}{l}\text { Neural descriptors generated by a graph neural } \\
\text { network pretrained with the small molecule dataset to } \\
\text { predict boiling temperature. }\end{array}$ \\
\hline $\left.\operatorname{Neural}(m p)^{c}\right)$ & 32 & Continuous & Neural descriptors for melting temperature. \\
\hline Neural(density) & 32 & Continuous & Neural descriptors for density. \\
\hline Neural(viscosity) & 32 & Continuous & Neural descriptors for viscosity. \\
\hline
\end{tabular}

a) Available as Descriptors.descList class in RDKit.

b) Available as CalcAUTOCORR3D, CalcMORSE, CalcRDF, and CalcWHIM classes in RDKit.

c) "bp" and " $m p$ " stands for boiling and melting temperatures, respectively.

Figure S2. Explanations of descriptors used in this study.

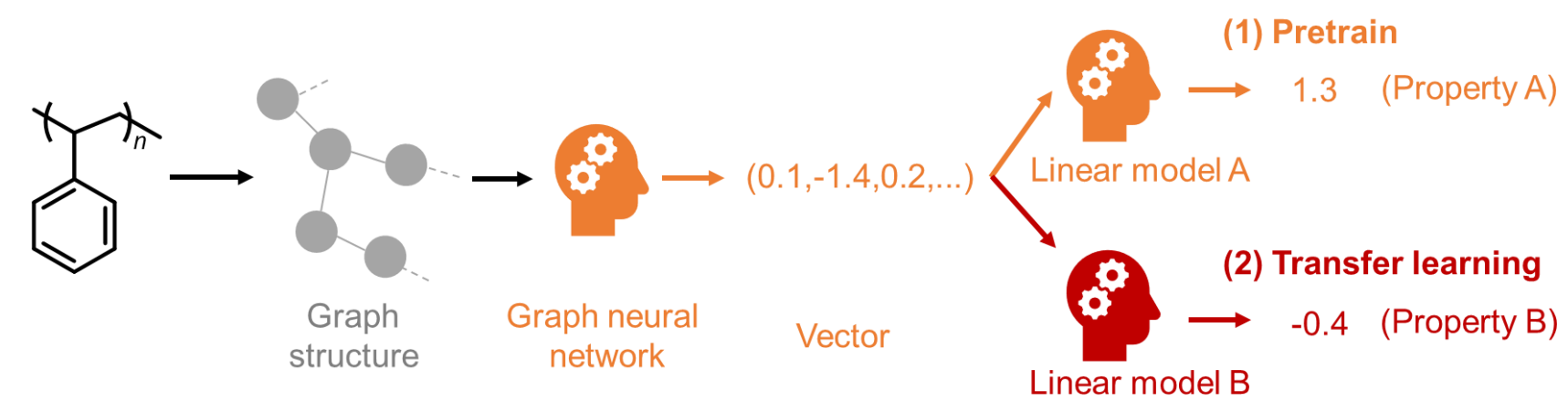

Figure S3. Scheme for transfer learning using graph neural networks. 


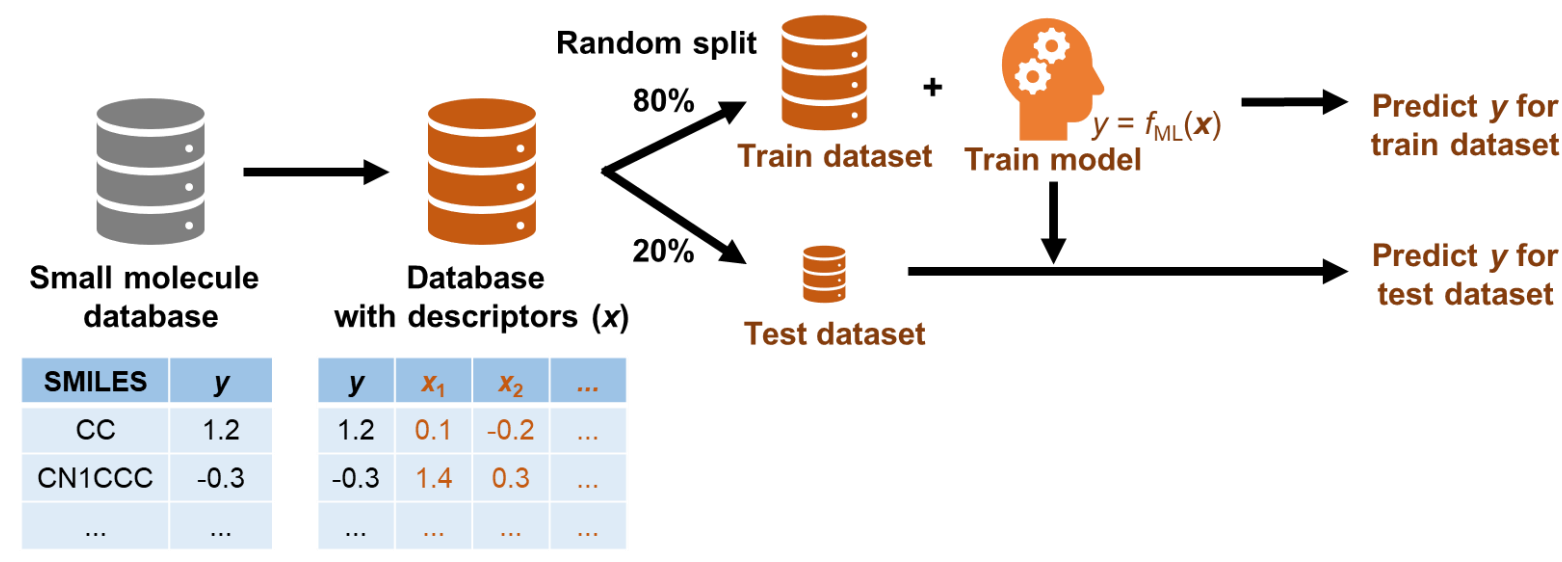

Figure S4. Scheme to predict molecular properties $(y)$ from their descriptors $(\boldsymbol{x})$.

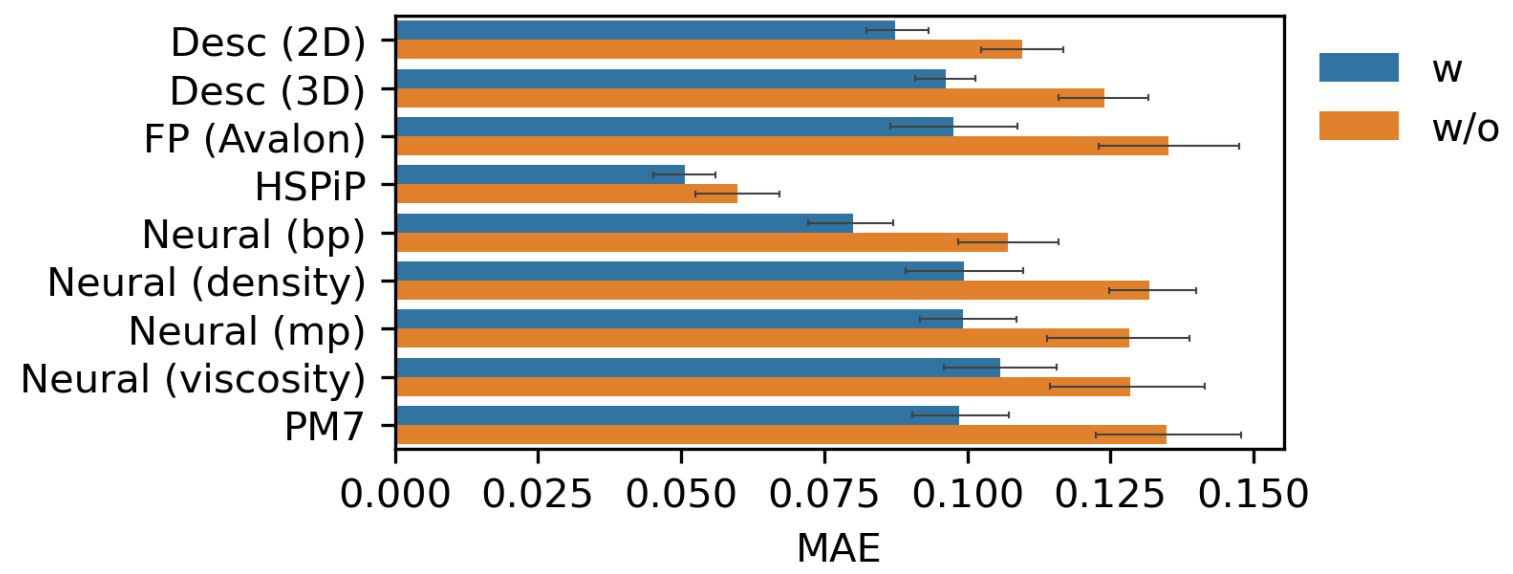

a)

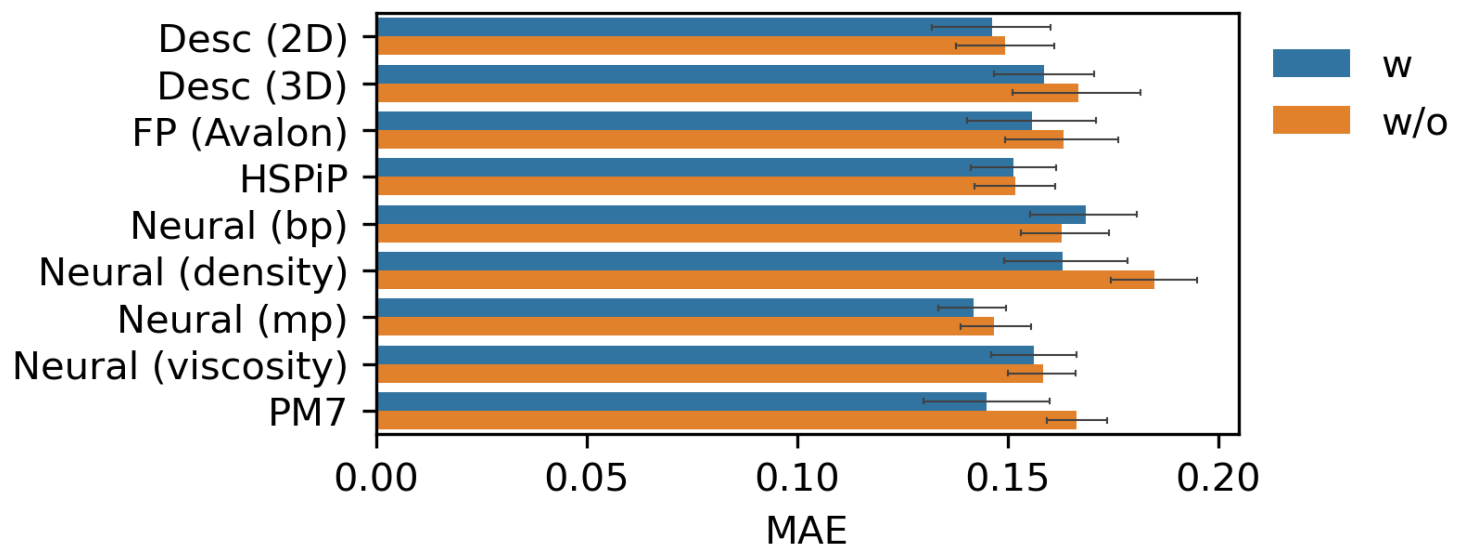

b) 


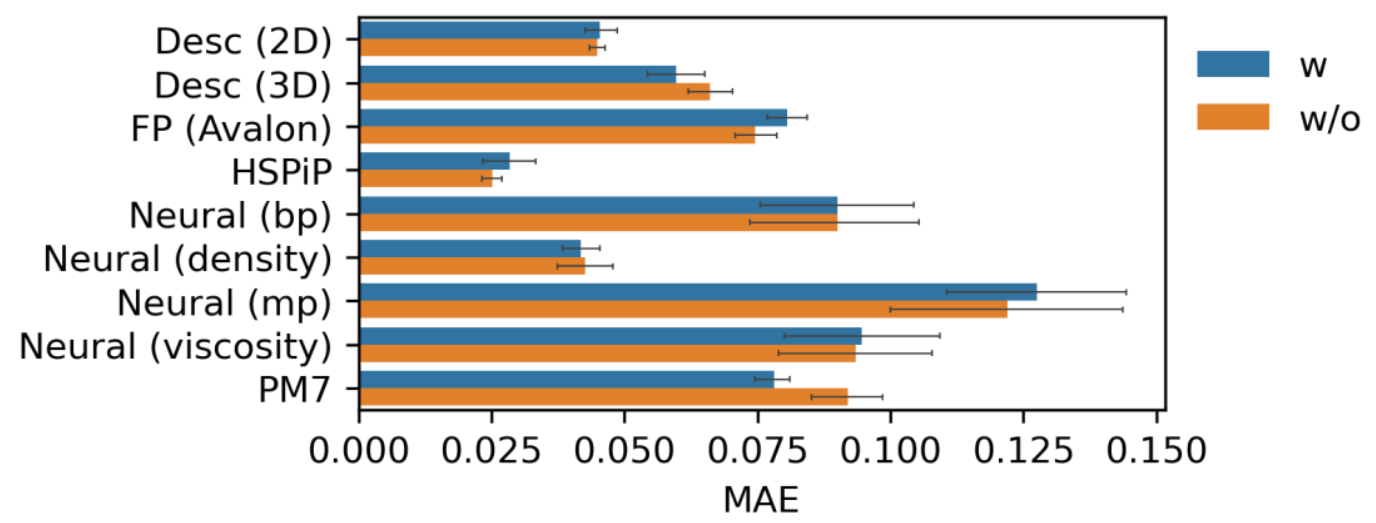

c)

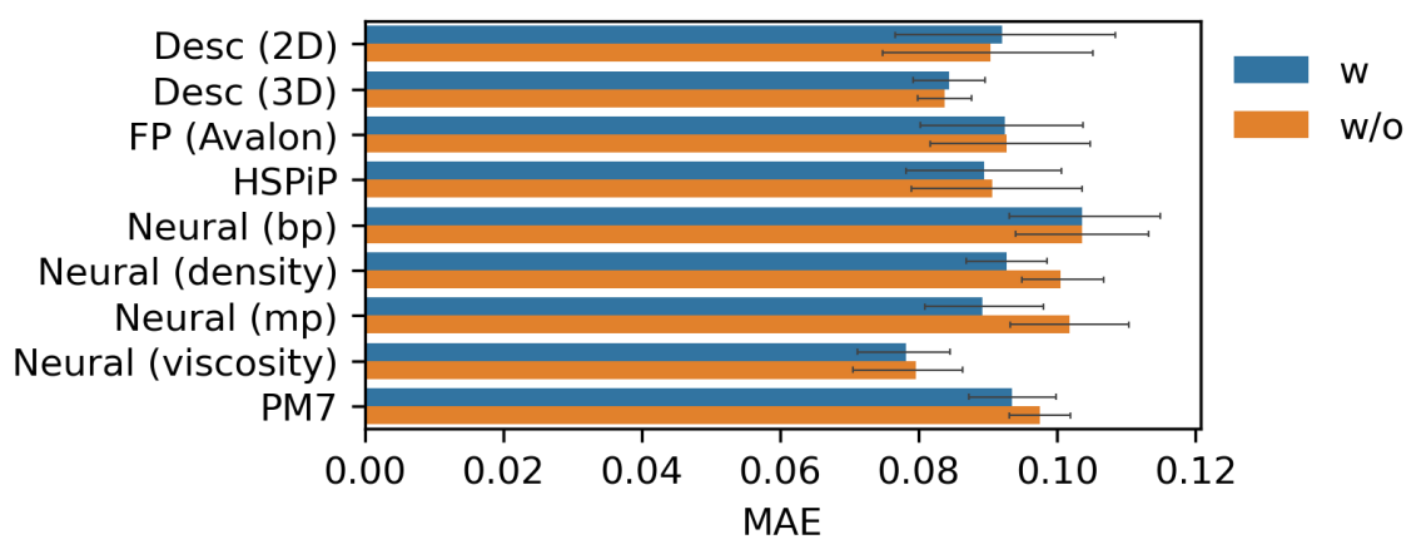

d)
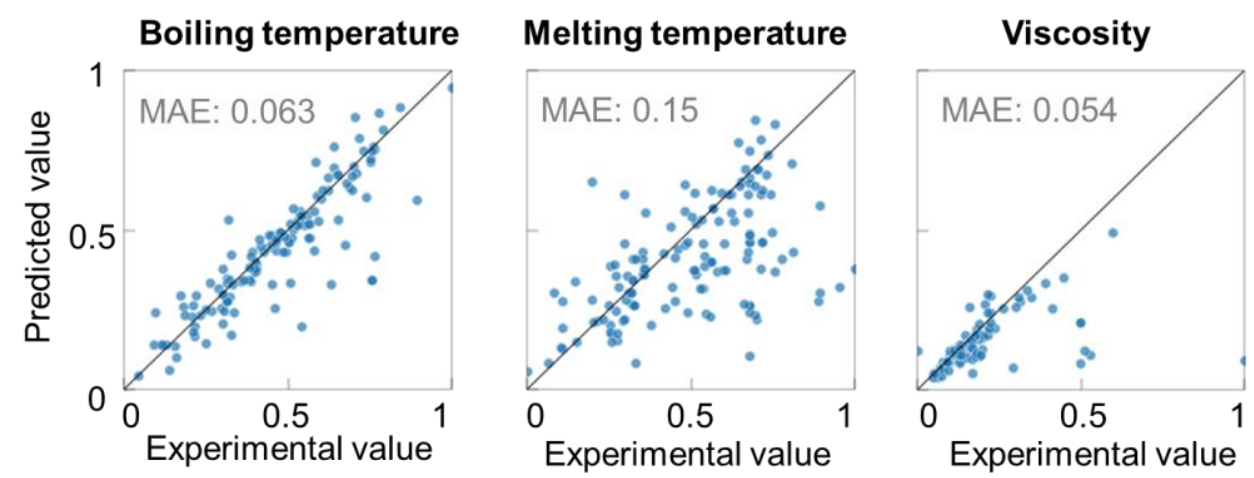

e)

Figure S5. Prediction results of a) boiling temperature, b) melting temperature, c) density, and d) viscosity. Average values of 5-hold cross-validation for test datasets are shown. Error bars represent standard errors. The legend " $w$ " indicates that experimental chemical properties (i.e., boiling temperature, melting temperature, density, and viscosity) were included in $\boldsymbol{x}$ in addition to original molecular descriptors, and "w/o" indicates not. XGB regressor was used as a predictor. e) Prediction of physical properties by a group contribution method, using a library of JRgui. ${ }^{1}$ 


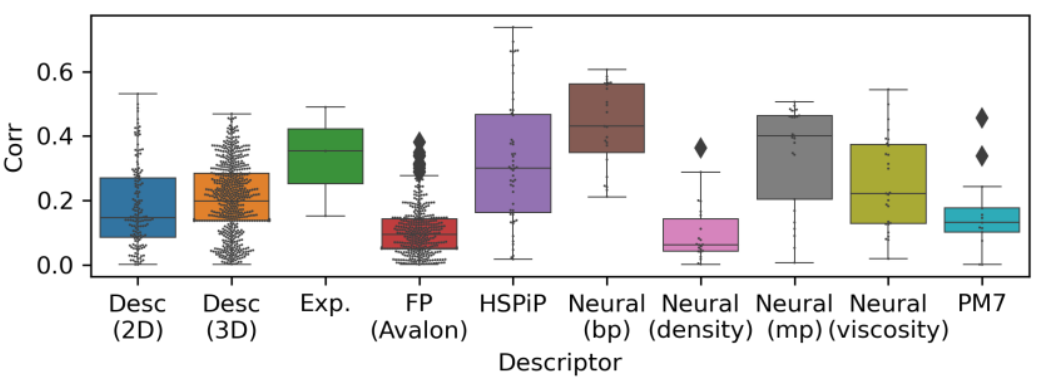

a)

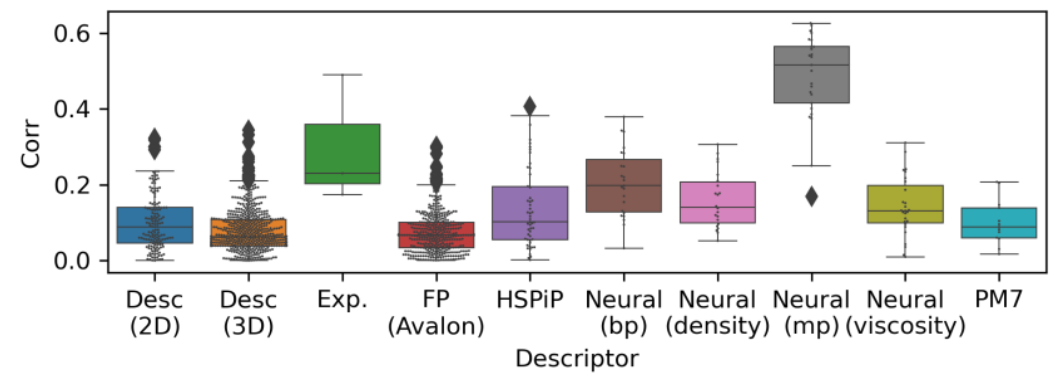

b)

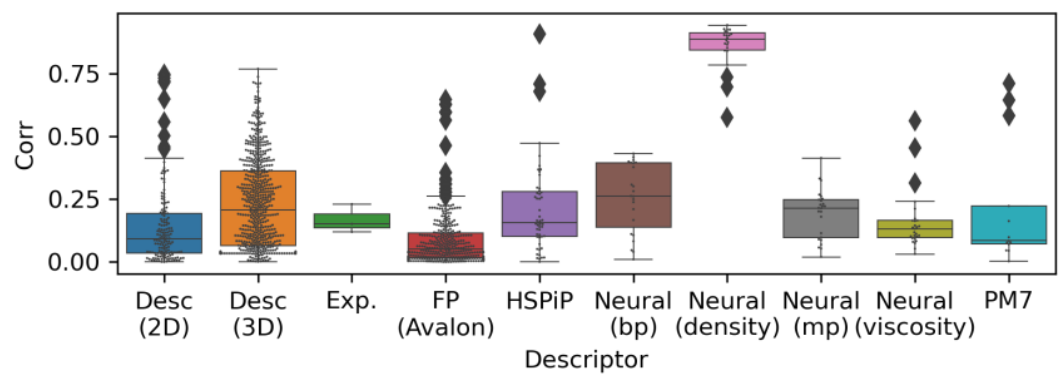

c)

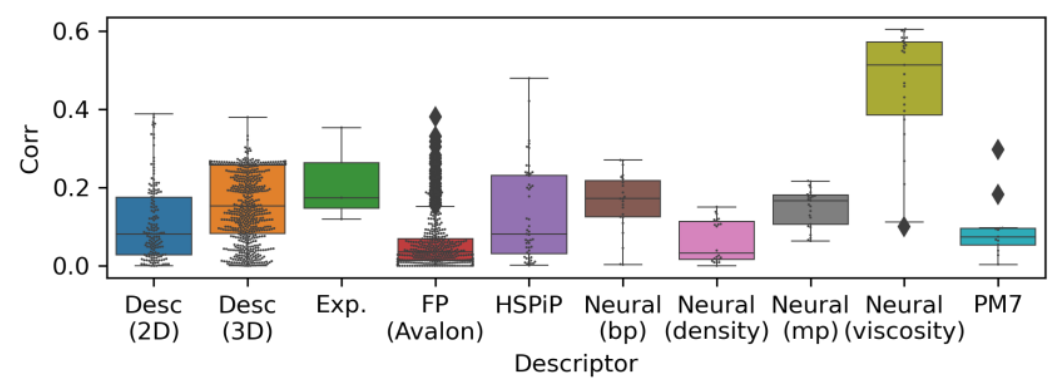

d)

Figure S6. Distribution of absolute correlation coefficients of molecular descriptors against a) boiling temperature, b) melting temperature, c) density, and d) viscosity. Exp, bp, and mp represent experimental chemical properties, boiling temperature, and melting temperature. 


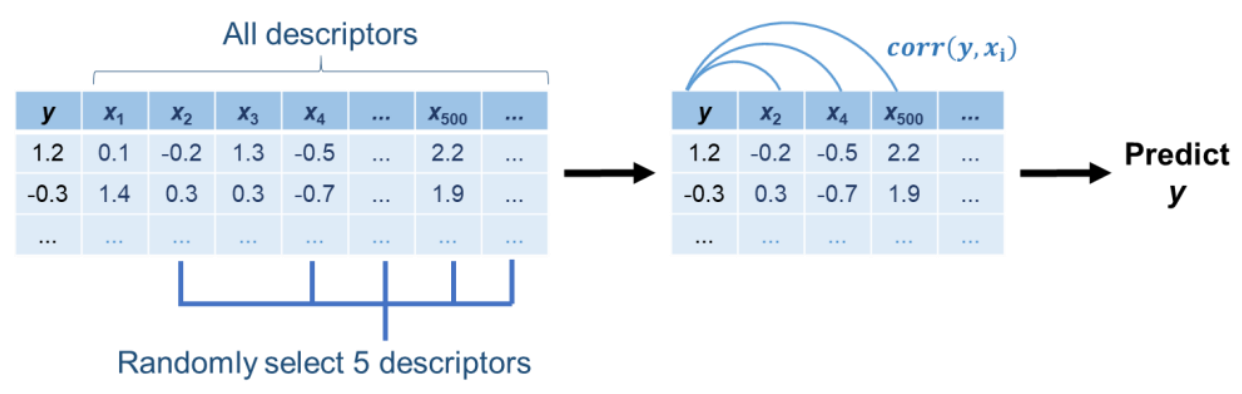

a)

\section{Extrapolation task}

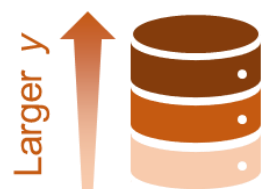

Database with descriptor (sorted by $y$ )

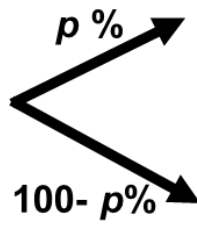

\section{ors}

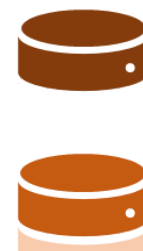

Train dataset
Test dataset

\section{Interpolation task}
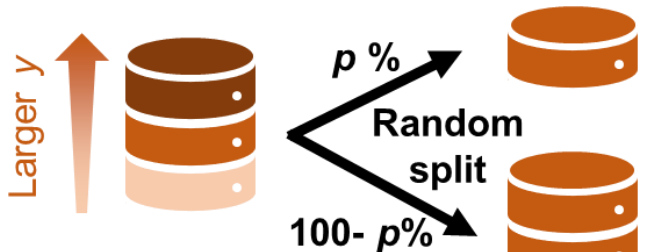

Test dataset

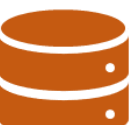

Train dataset

b)

Figure S7. a) Scheme to predict $y$ from randomly selected 5 descriptors. The function $\operatorname{corr}\left(y, x_{\mathrm{i}}\right)$ indicates the absolute value of the correlation coefficient between y and $x_{\mathrm{i}}$. Averages of $\operatorname{corr}\left(y, x_{\mathrm{i}}\right)$ for the selected 5 descriptors are used as the $x$-axis in Figure S8. b) Comparison of the extrapolation and interpolation tasks. For the extrapolation, top $p \%$ records of $y$ were used for a test dataset $(p=20)$. 


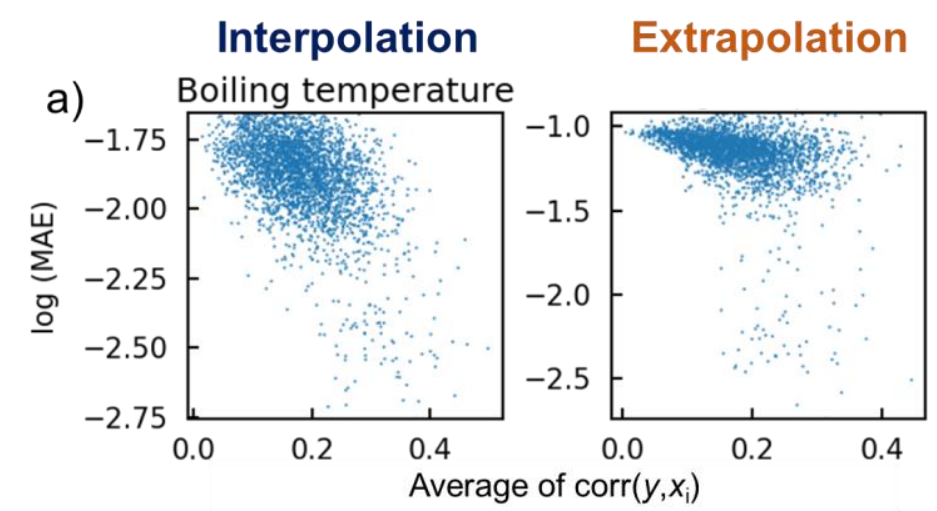

b) Melting temperature
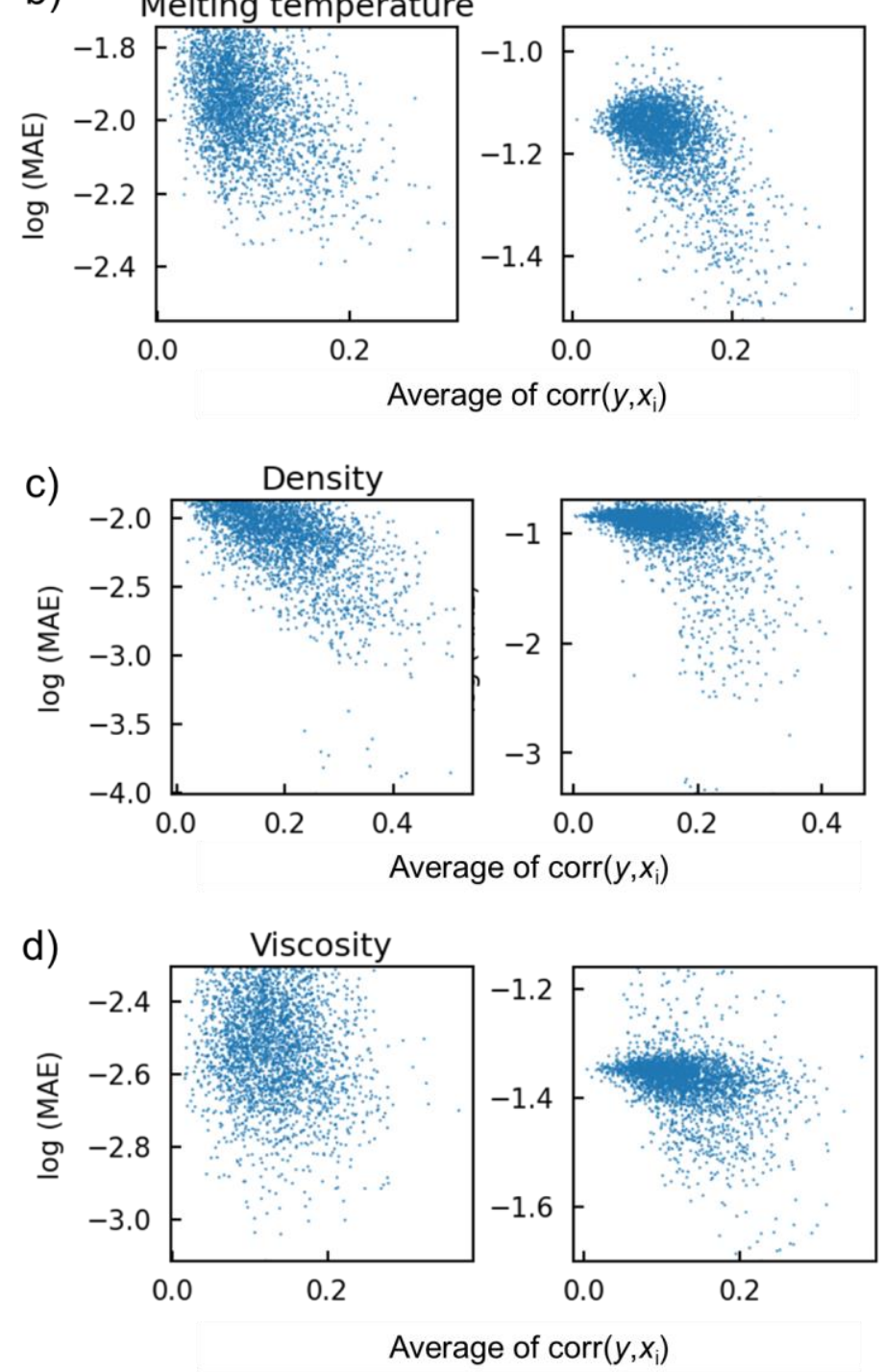

Figure S8. Relationship between MAE and average of $\operatorname{corr}\left(y, x_{\mathrm{i}}\right)$ for a) boiling temperature, b) melting temperature, c) density, and d) viscosity prediction. Datasets were prepared according to a rule shown in Figure S7. Huber and XGB regressors were used for interpolation and extrapolation tasks, respectively. 
Interpolation

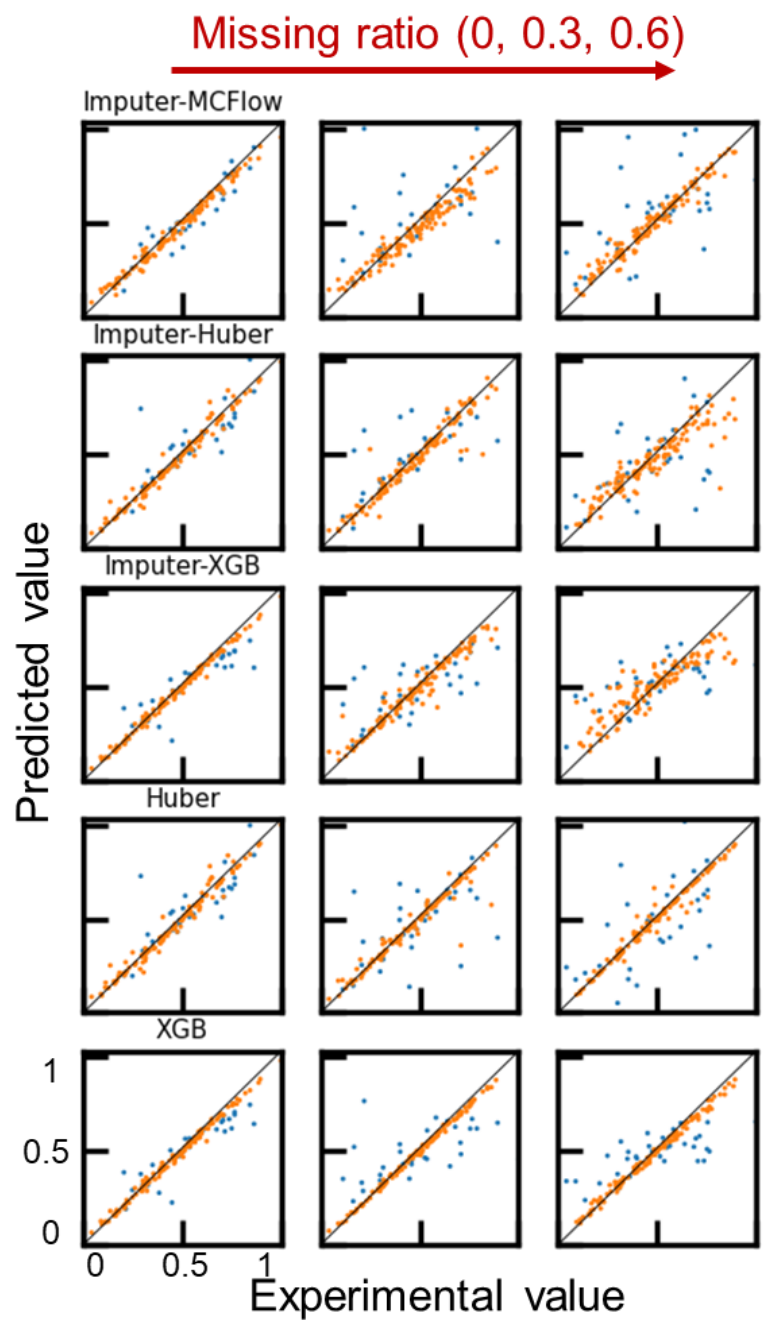

Extrapolation

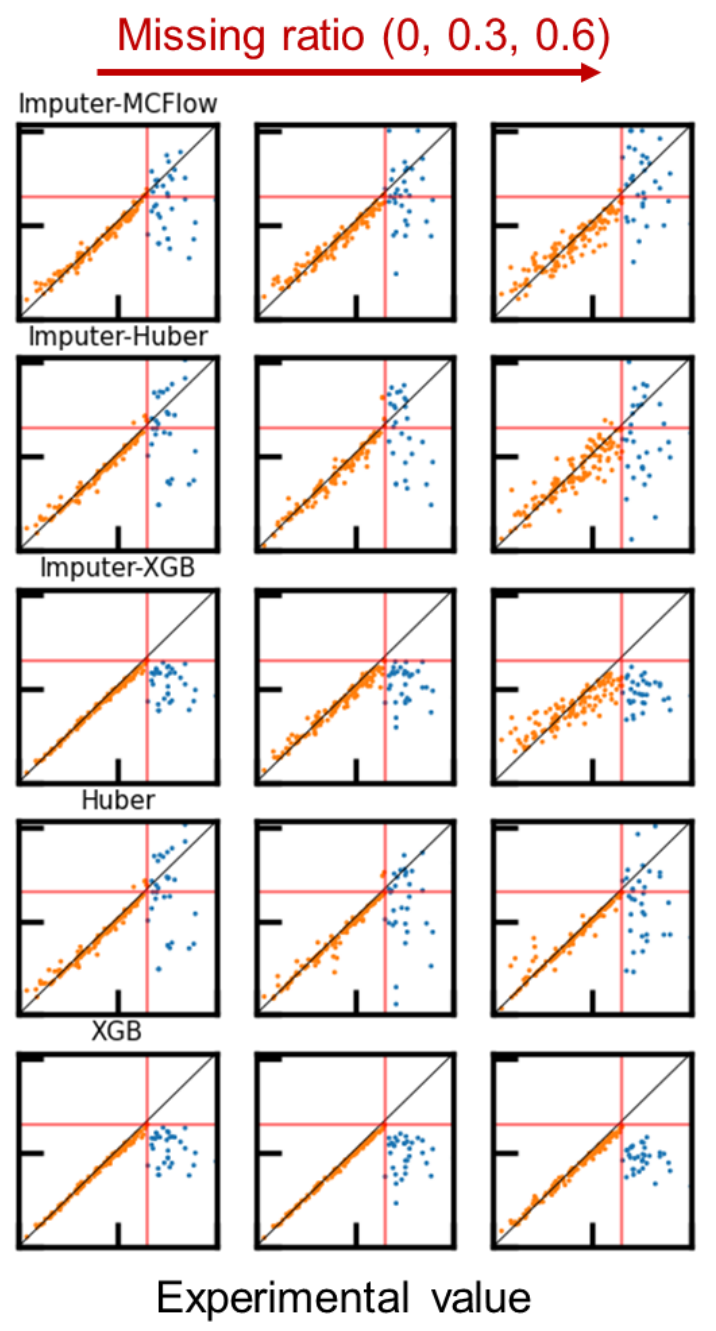

Boiling temperature

a) 


\section{Interpolation}

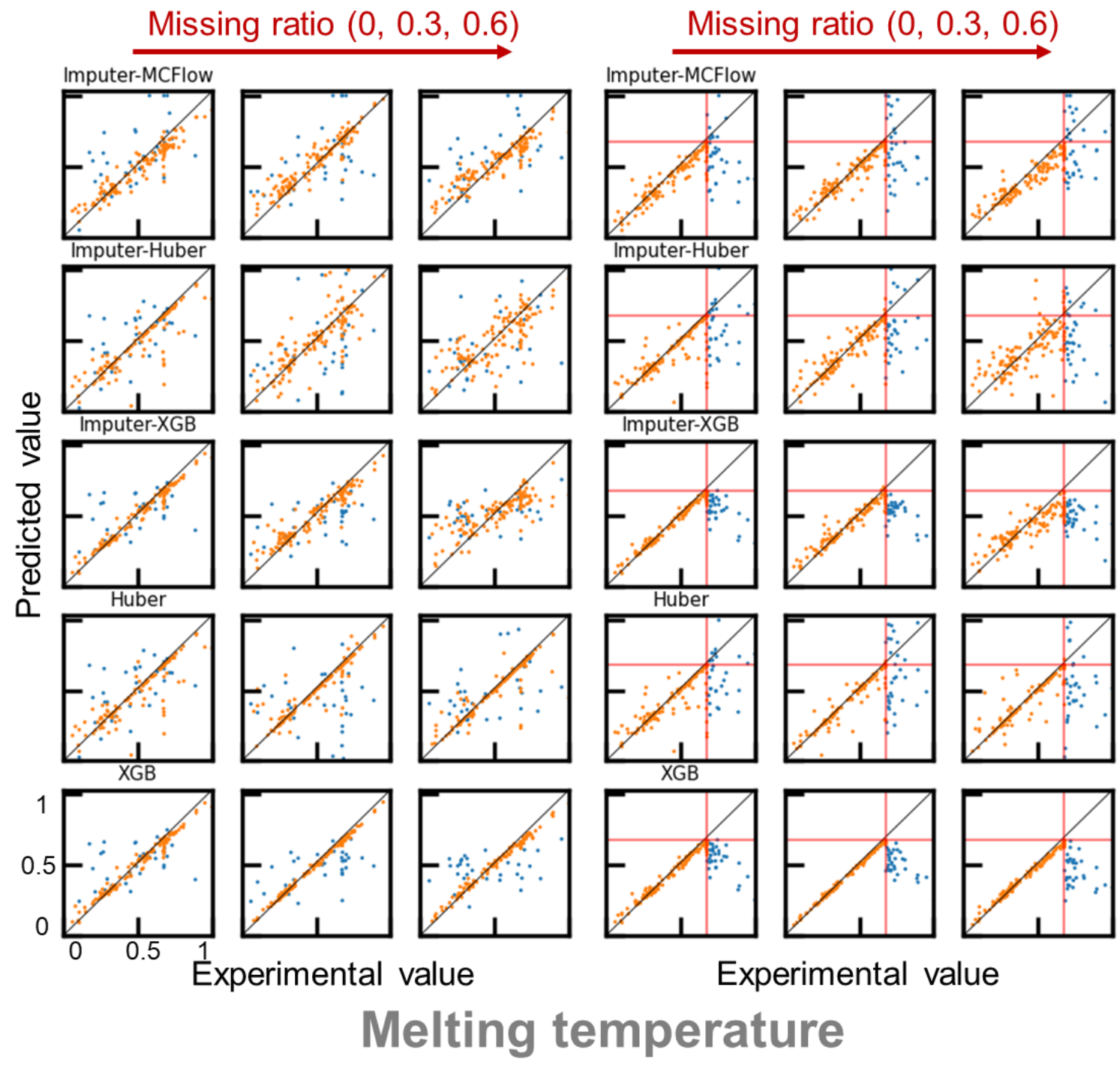

b) 


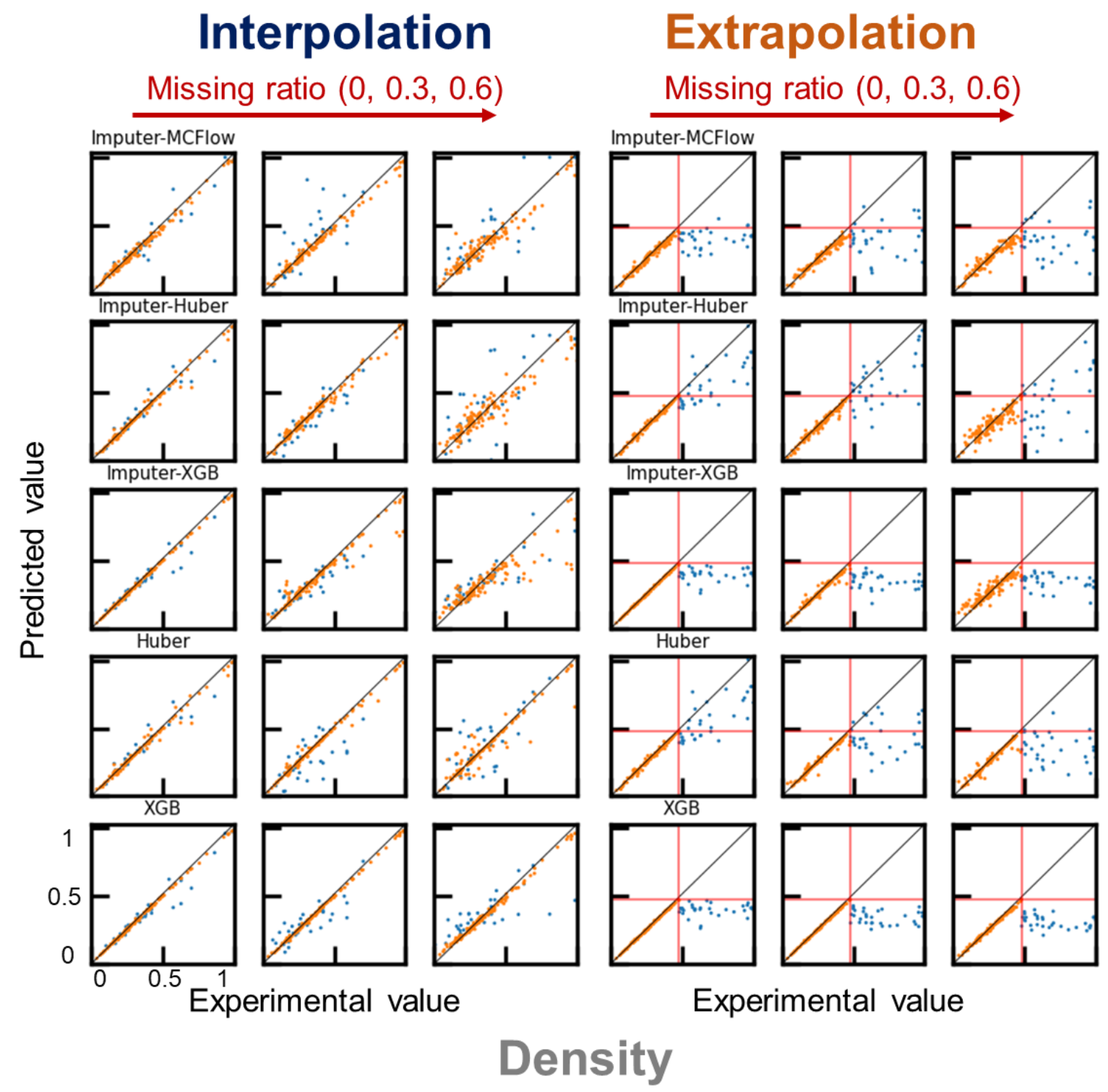

c) 


\section{Interpolation}

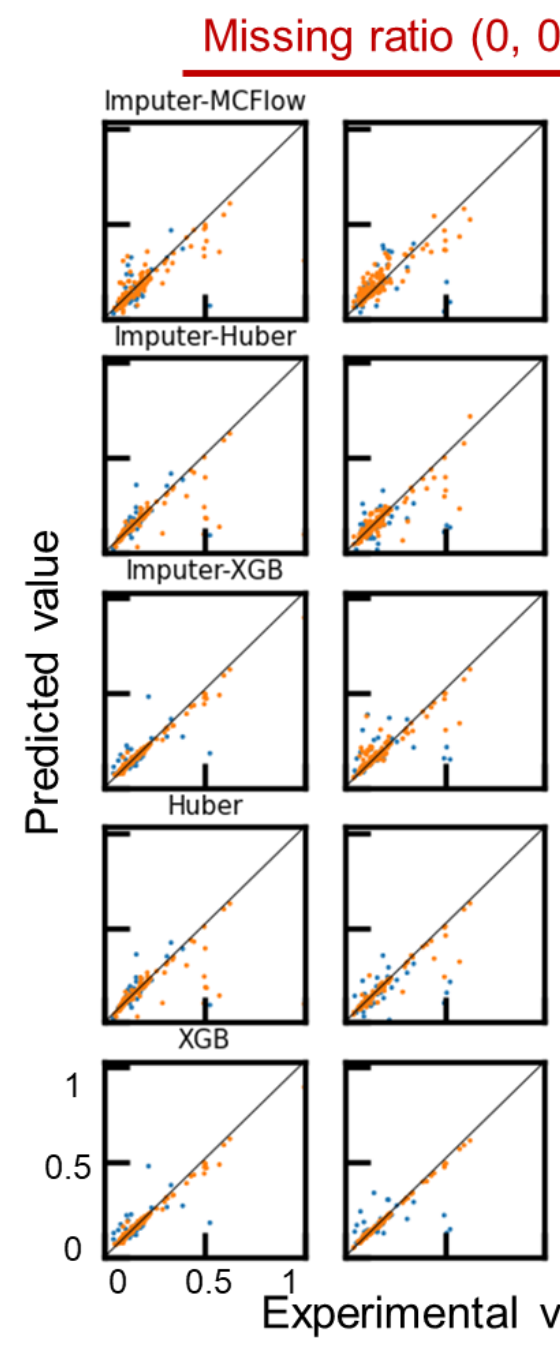

\section{Extrapolation}
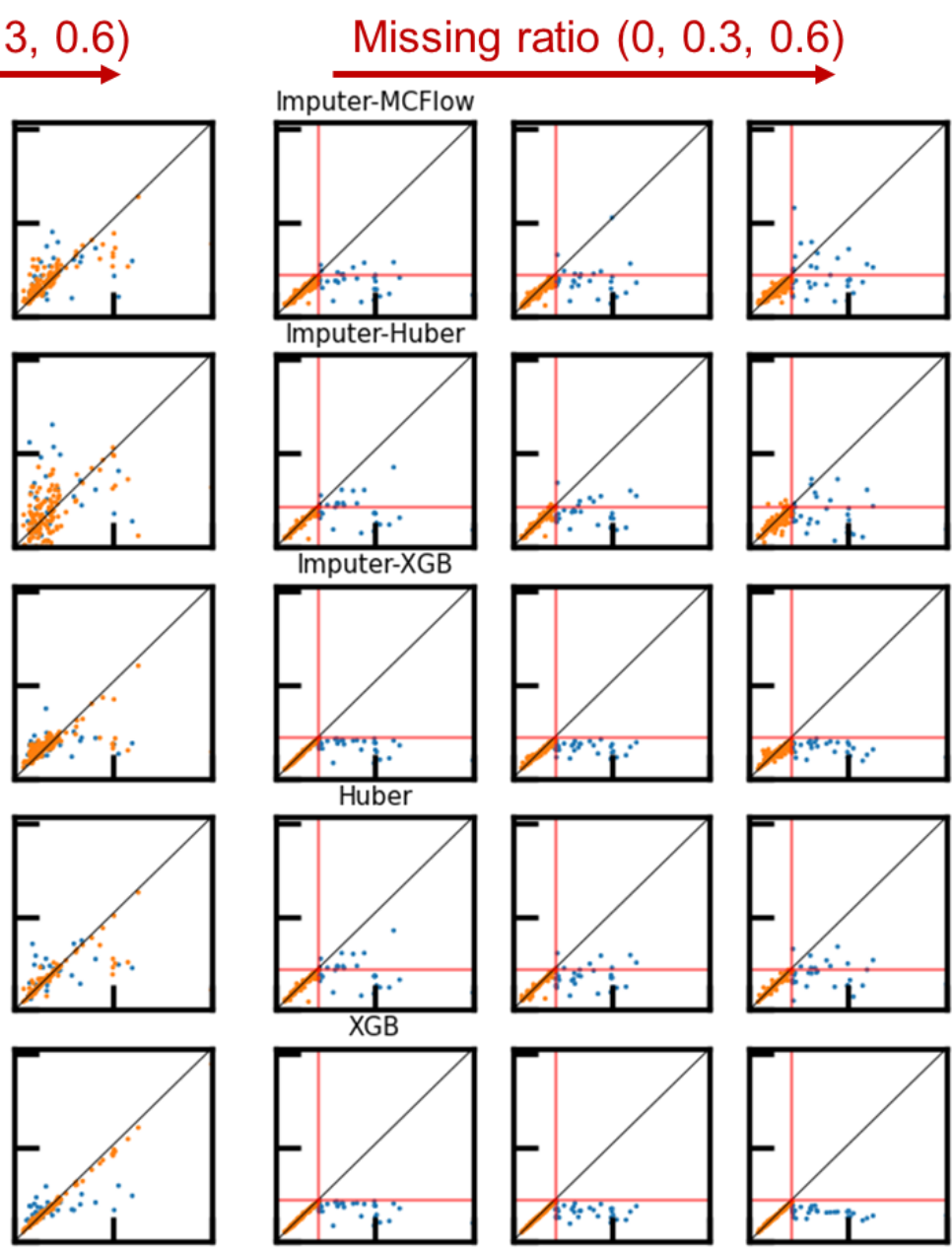

Experimental value
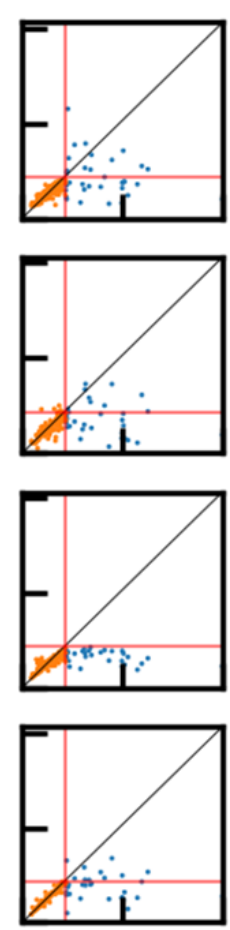

Viscosity

d)
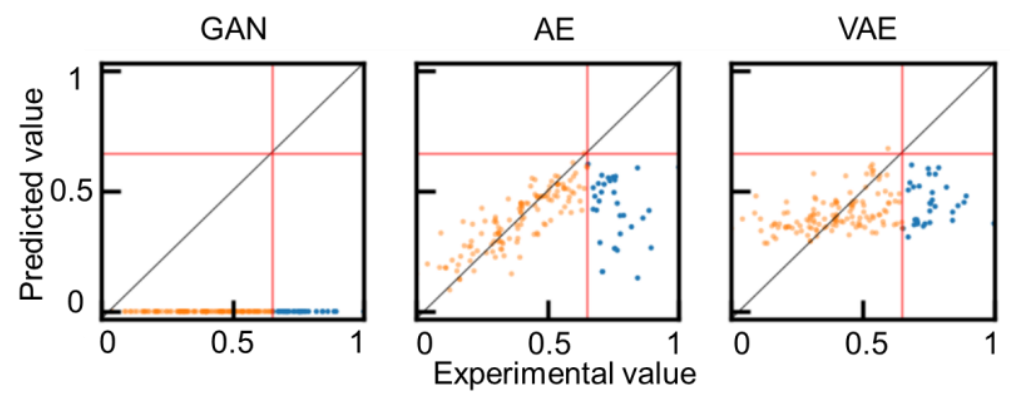

e) 
Figure S9. Prediction results of a) boiling temperature, b) melting temperature, c) density, and d) viscosity, using different algorithms. Orange and blue plots represent train and test datasets, respectively. Before regression, $p(=0,30$, or 60$) \%$ of explanatory variables $(\boldsymbol{x})$ were filled with missing values $(\mathrm{n} / \mathrm{a})$ randomly. Imputers predicted $y$ by imputing the missing values. Mean values of $x_{\mathrm{i}}$ were used instead of the missing values for the Huber regressor. XGB regressor could input missing values directly for prediction. e) Extrapolating prediction results by GAN, AE, and VAE. Boiling temperature was predicted without data missing.

Interpolation

a)

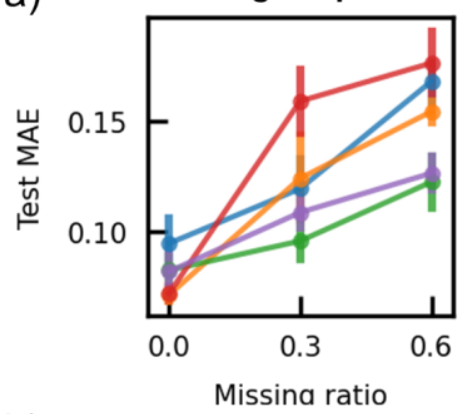

b)

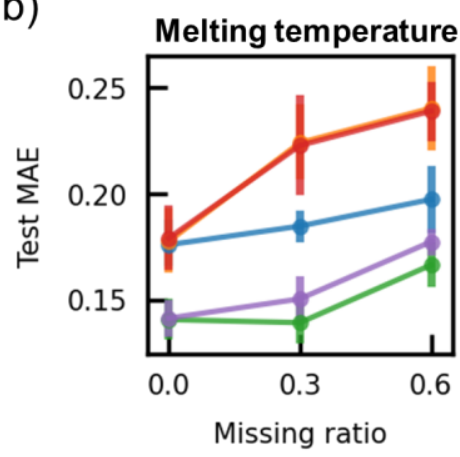

c)

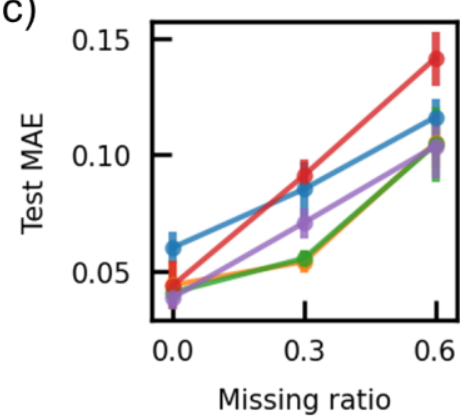

d)

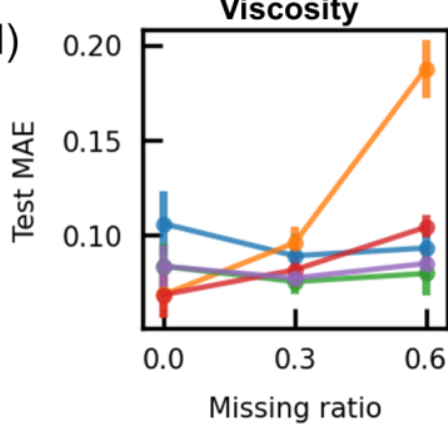

\section{Extrapolation}
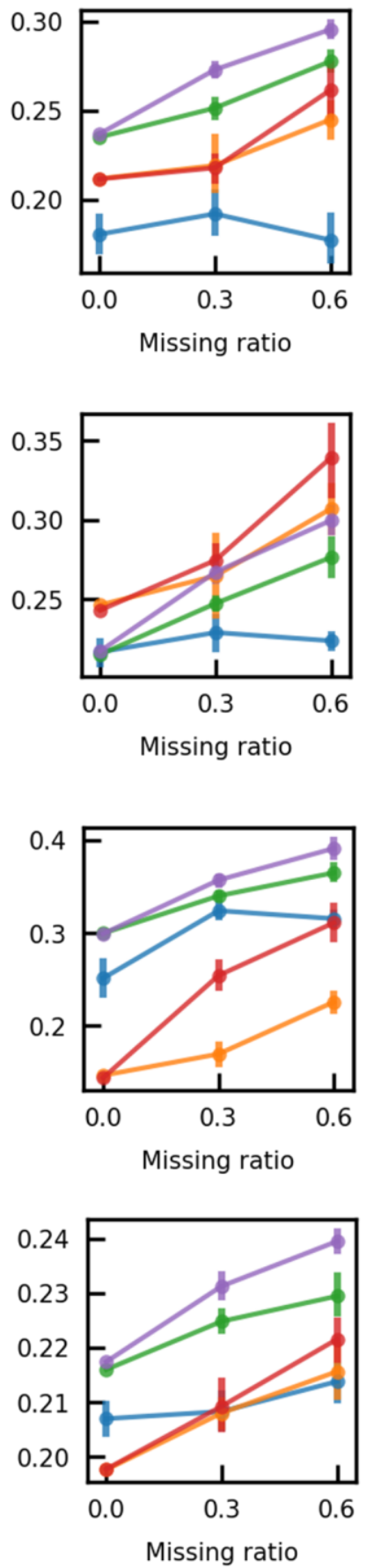


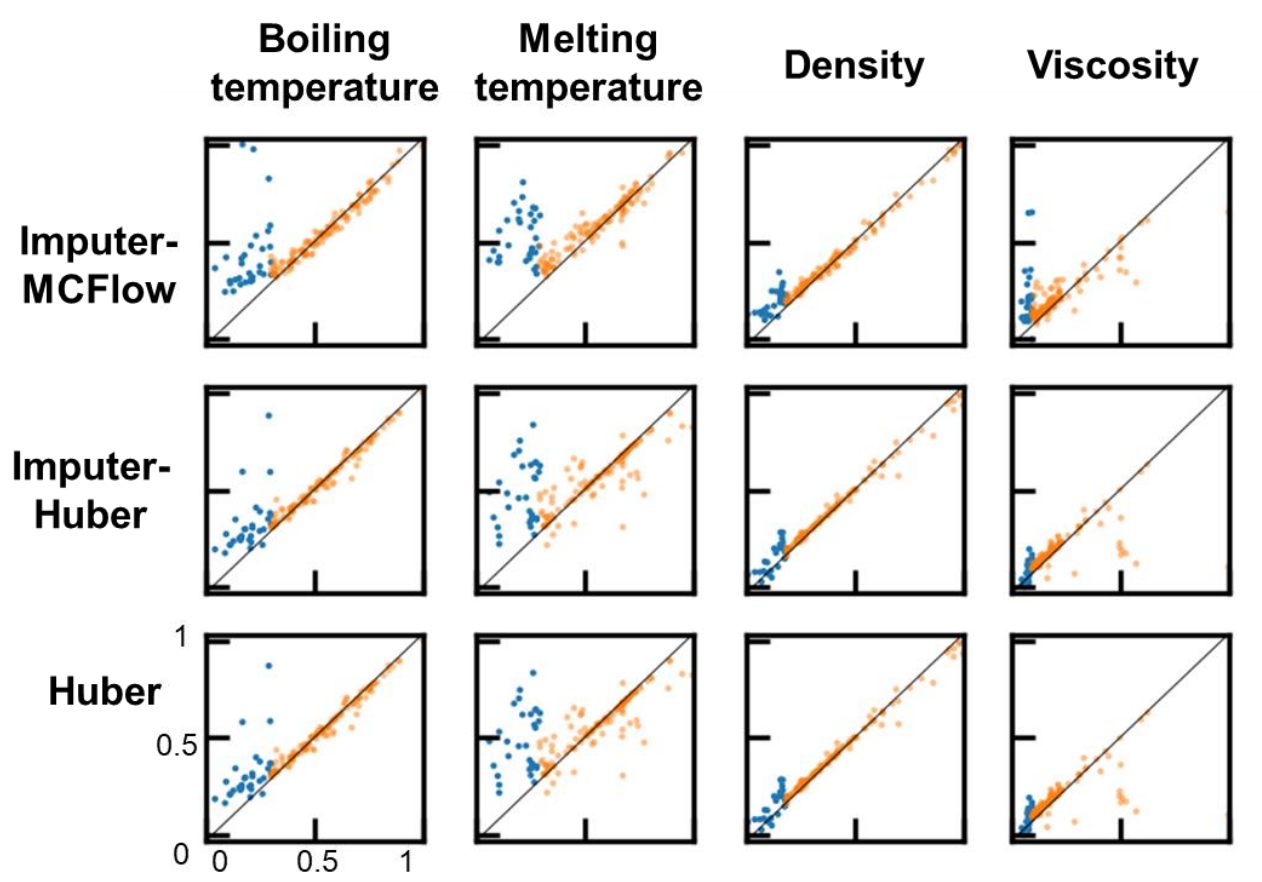

e)

\section{Extrapolation (inverse)}

\section{Boiling temperature Melting temperature}
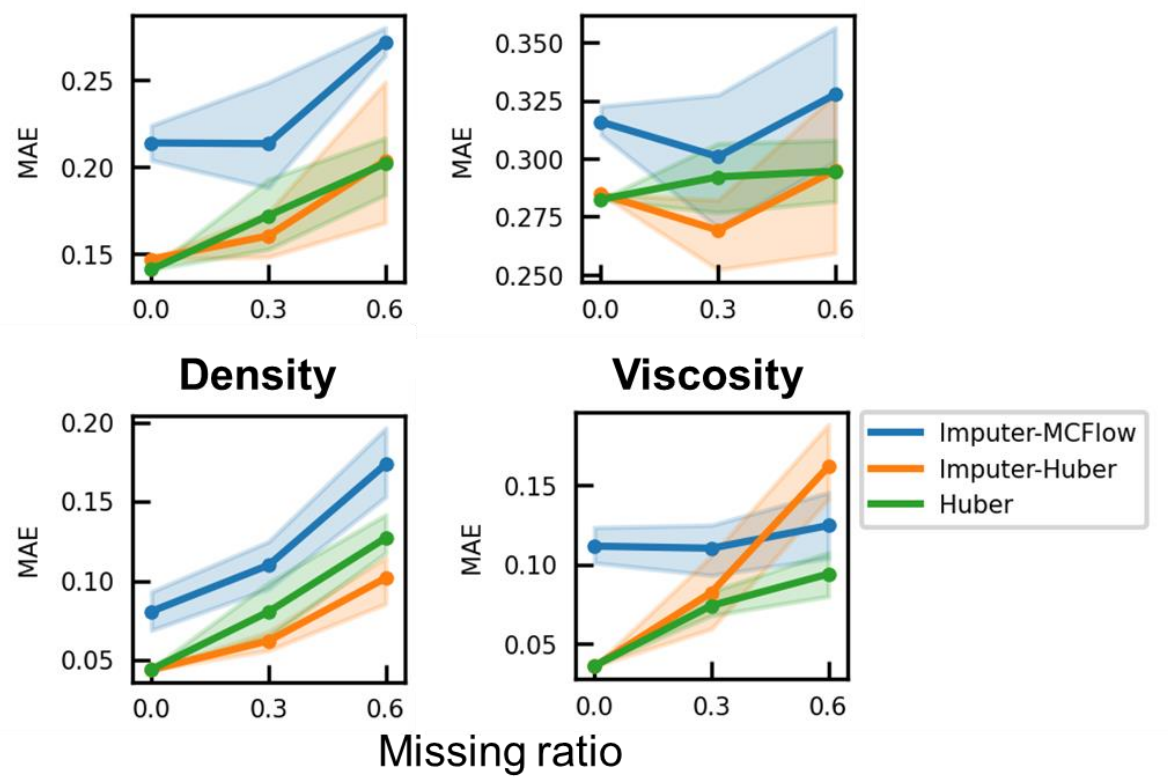

f)

Figure S10. a-d) Average MAE for the prediction tasks shown in Figure S9. Results for the test data are presented. Dataset preparation and prediction were repeated 5 times. Error bars show standard errors. e) Extrapolation prediction using the testing data with the lowest $20 \%$ targeting parameters $(y)$. Representative data with the missing ratio of $p=0$ are shown. f) Average MAEs for the prediction tasks of e). 


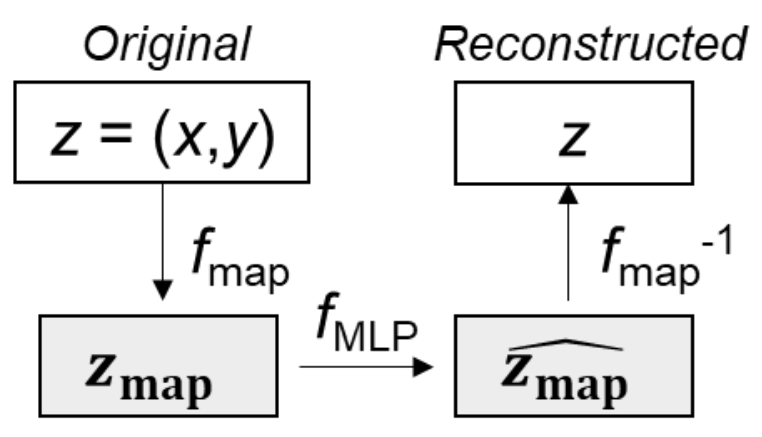

a)

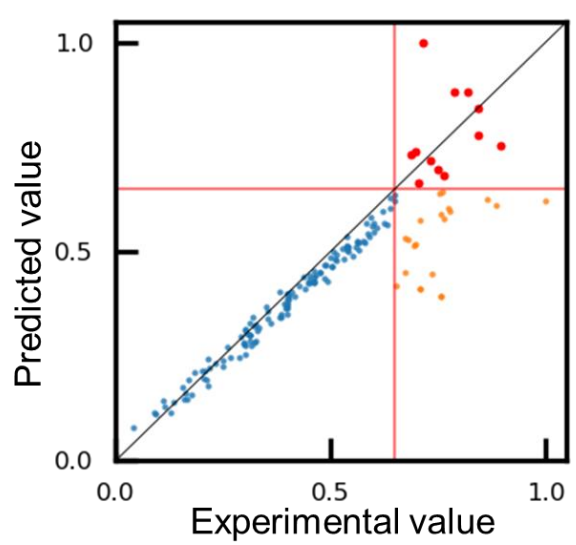

b)
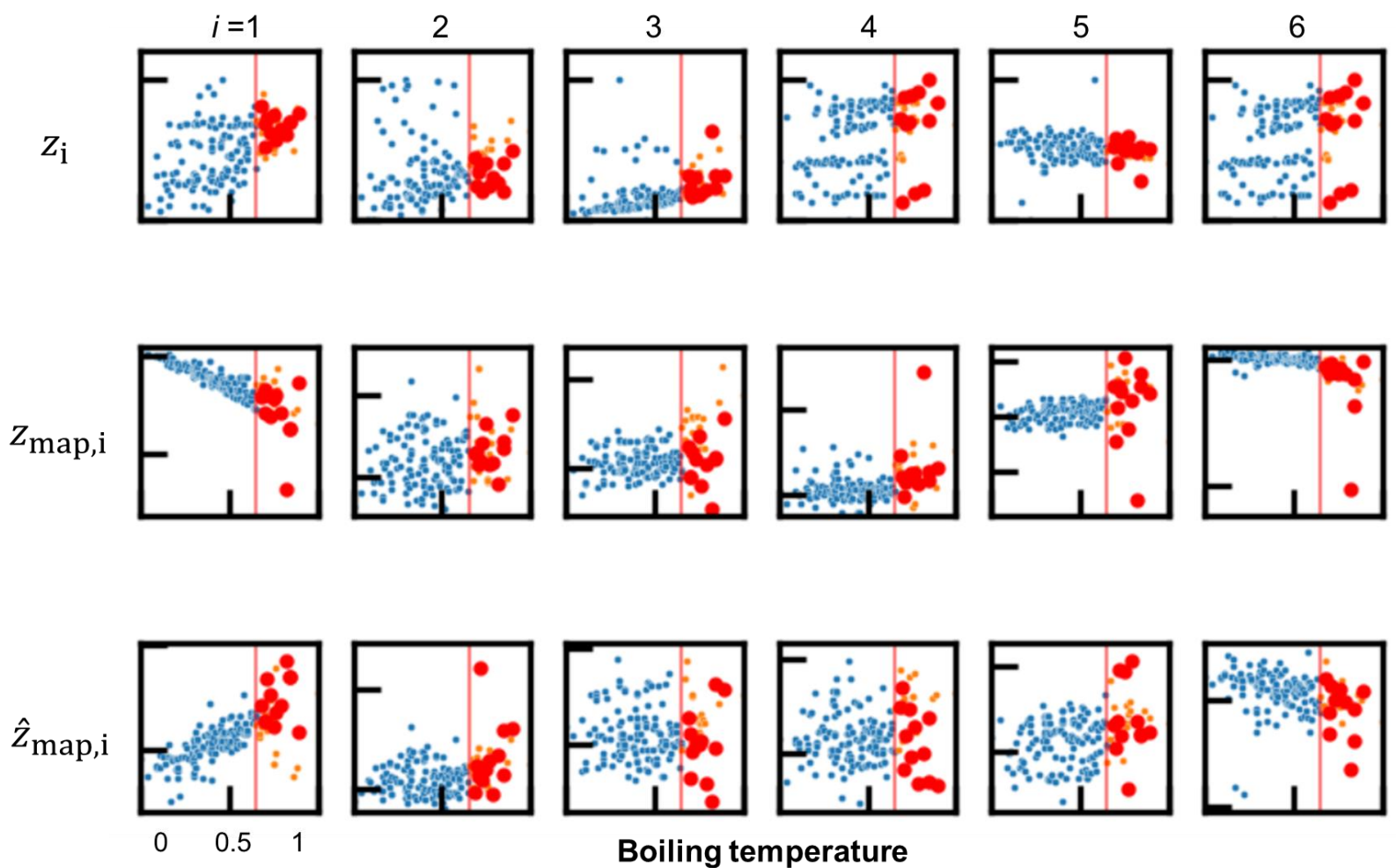

c) 


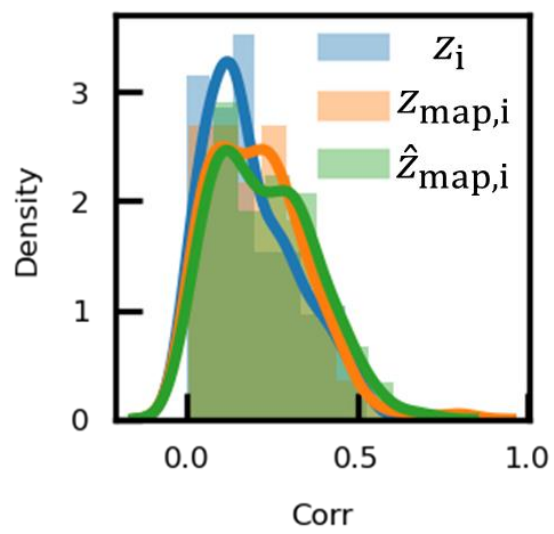

d)

Figure S11. a) Scheme of MCFlow. Original data $(x, y)$ was first converted into $\boldsymbol{z}_{\text {map }}$ having the same dimension as the original by $f_{\text {map }}$. Then, it was converted into $\hat{\mathbf{z}}_{\text {map }}$ by a multilayer perceptron function $f_{\text {MLP }}$, and into the original space $(x, y)$ by $f_{\text {map }^{-1}}$. The detailed implementation and source code are available at GitHub (https://github.com/KanHatakeyama/gen_model). b) Prediction result of boiling temperature (same task as Figure S9 without data missing) by the model. c) Representative $\boldsymbol{z}, \boldsymbol{z}_{\text {map }}$, and $\hat{\boldsymbol{z}}_{\text {map }}$ as a function of boiling temperature $(y)$. d) Distribution of absolute correlation coefficients between the variables and $y$. If predicted values exceeded that of the maximum of trained $y$, they were marked by red. The model was trained to predict boiling temperature from the small compound database without data missing (i.e., the same task as Figure S10a). 


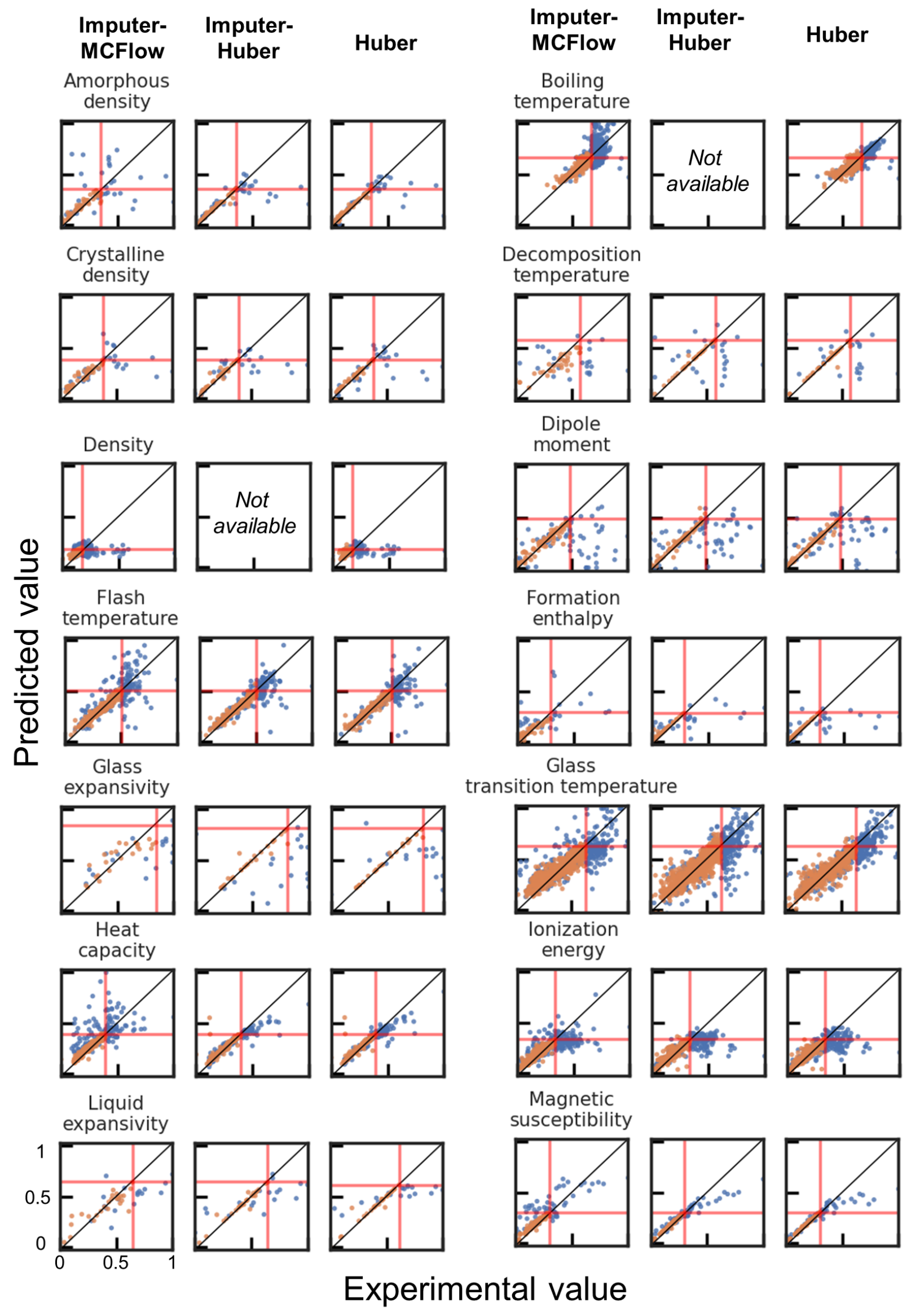




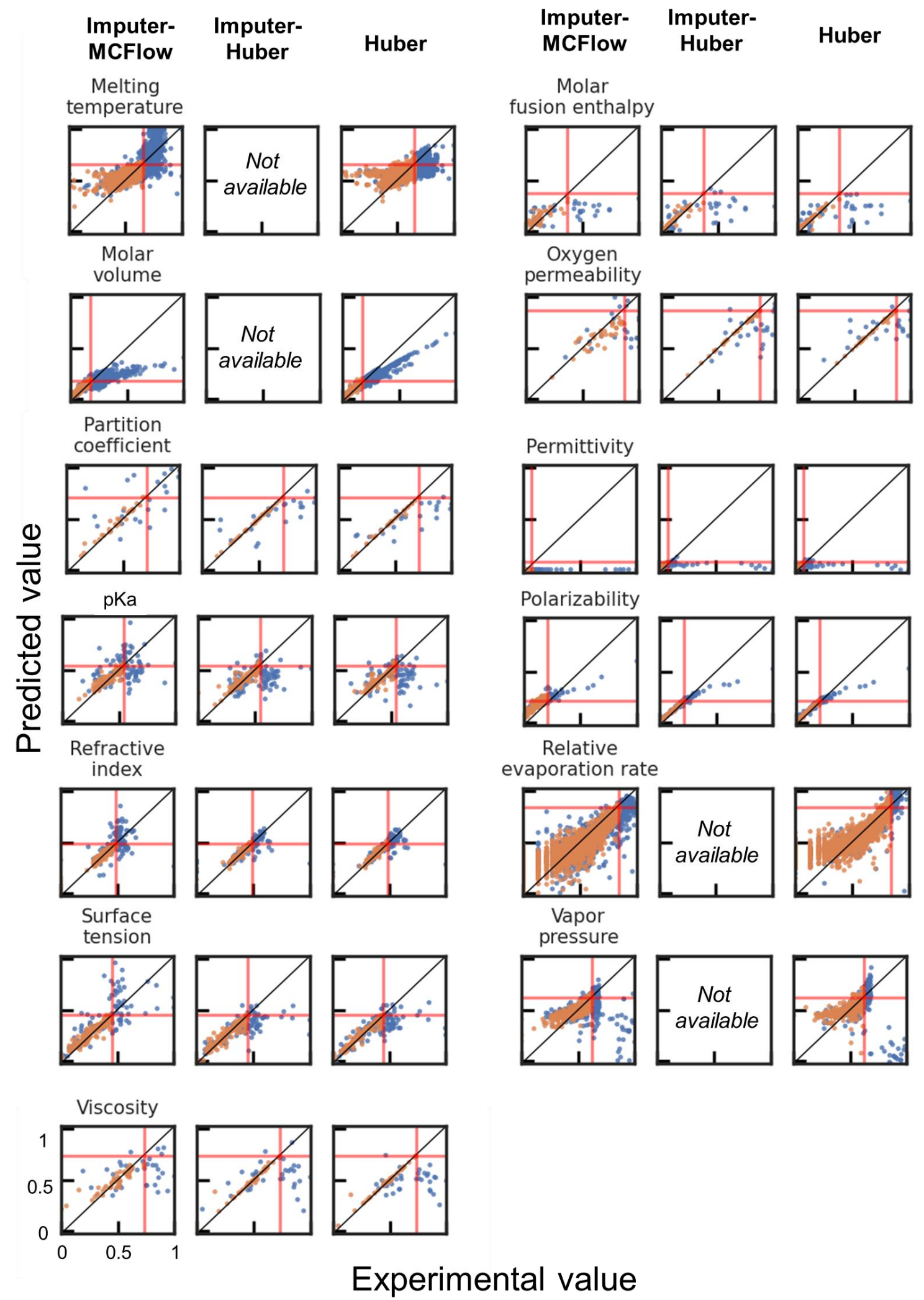

Figure S12. Prediction of various parameters recorded in the integrated database. Orange and blue plots represent the randomly selected $80 \%$ train and $20 \%$ test data, respectively. Some results by Imputer-Huber are not shown because of the high calculation cost of the model (e.g., the calculation will take more than 100 hours with properties containing over 10000 records). 
Amorphous density

Boiling temperature

Crystalline density

Decomposition temperature

Density

Dipole moment

Flash temperature

Formation enthalpy

Glass expansivity

Glass transition temperature

Heat capacity

Ionization energy

Liquid expansivity

충

Magnetic susceptibility

Melting temperature

Molar fusion enthalpy

Molar volume

Oxygen permeability

Partition coefficient

Permittivity

$\mathrm{pKa}$

Polarizability

Refractive index

Relative evaporation rate

Surface tension

Vapor pressure

Viscosity
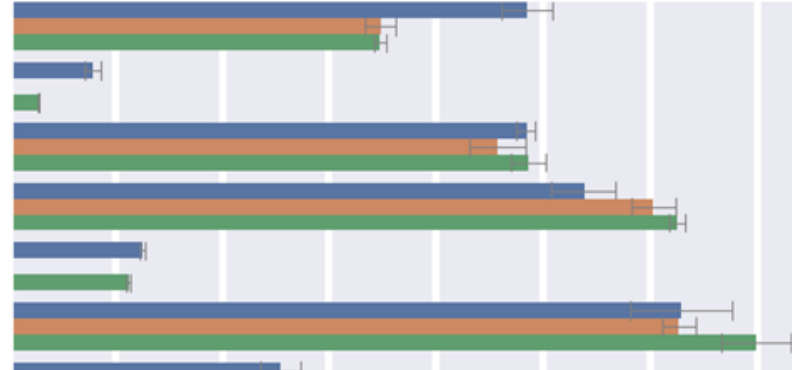

a
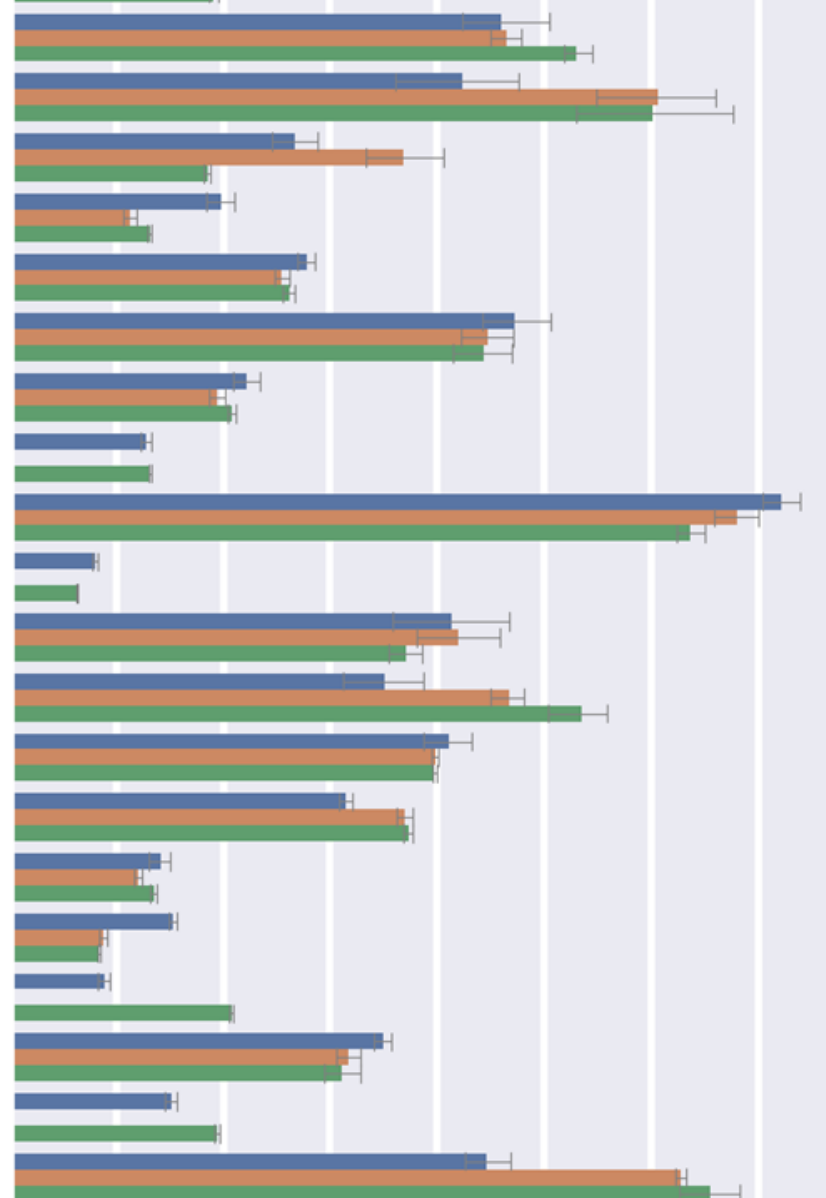

0.00

0.05

0.10

0.15

0.2

0.25

0.30

0.35

MAE
Imputer-MCFlow

Imputer-Huber

Huber 
Boiling temperature

Crystalline density

Decomposition temperature

Density

Dipole moment

Flash temperature

Formation enthalpy

Glass expansivity

Glass transition temperature

Heat capacity

Ionization energy

Liquid expansivity

충

Magnetic susceptibility

Melting temperature

Molar fusion enthalpy

Molar volume

Oxygen permeability

Partition coefficient

Permittivity

$\mathrm{pKa}$

Polarizability

Refractive index

Relative evaporation rate

Surface tension

Vapor pressure

Viscosity
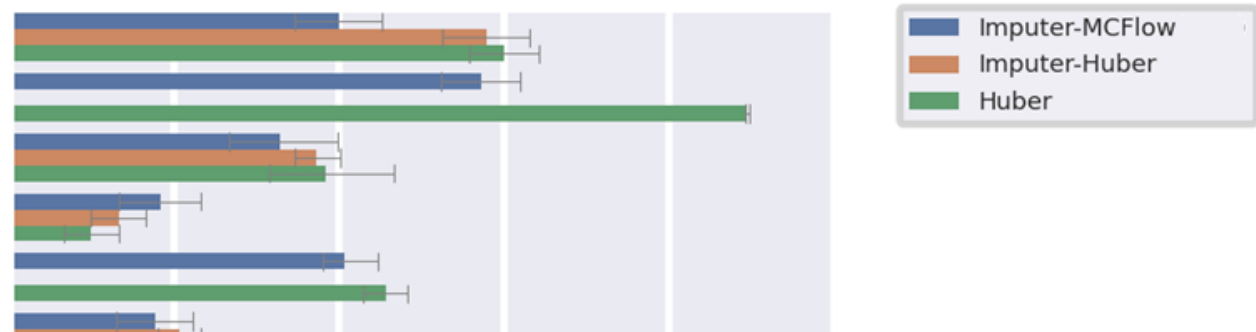

넌
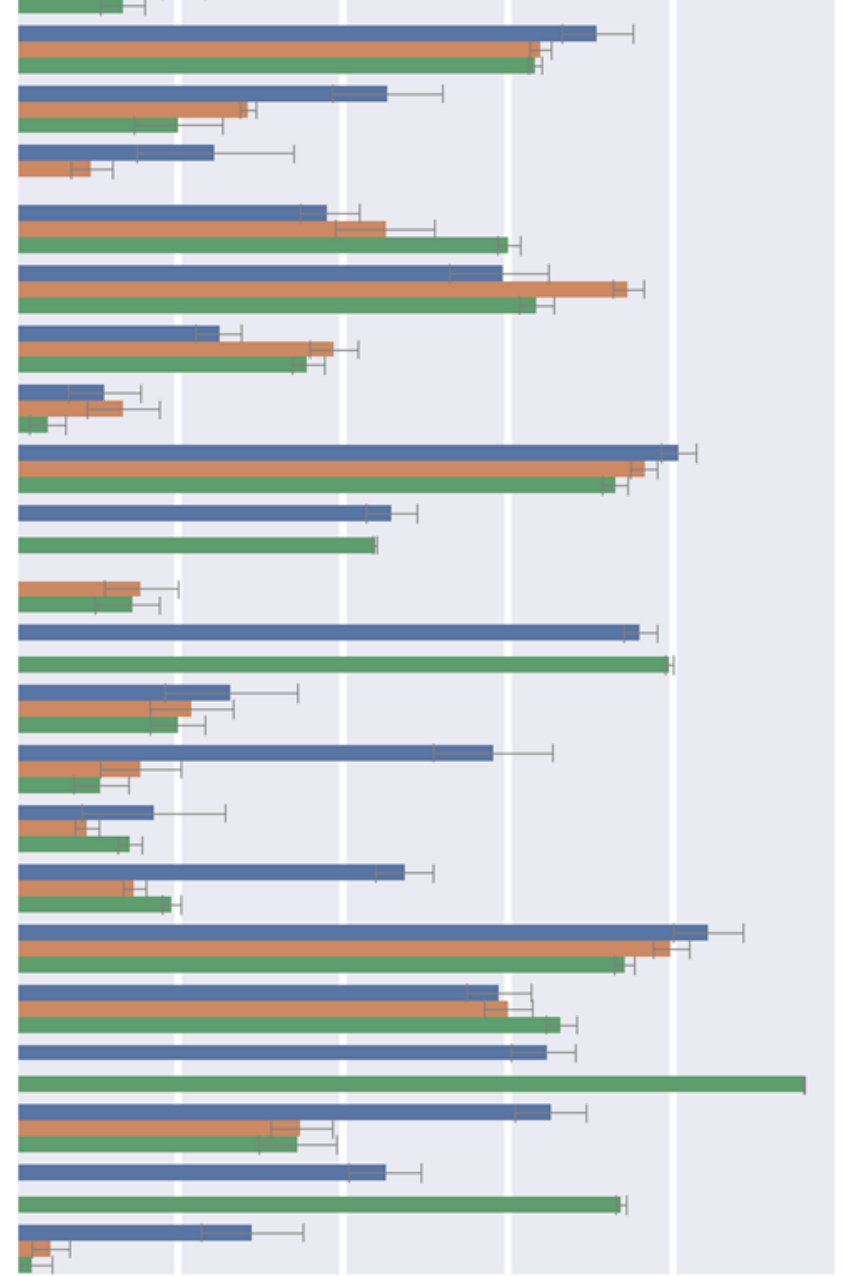

20

40

60

80

100

Recall (\%)

b) 


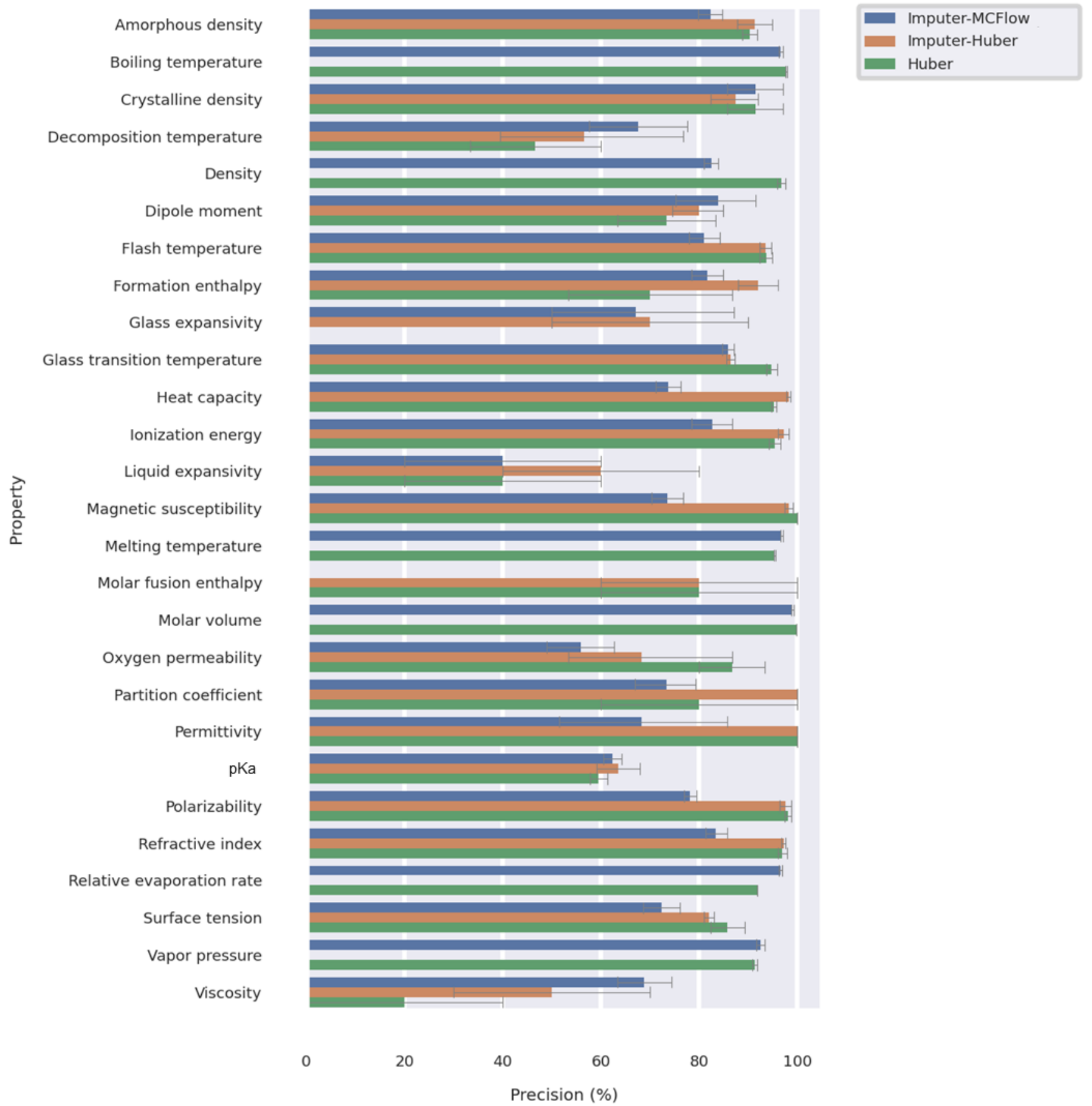

c)

Figure S13. Prediction performances for the test datasets of the integrated database. a) MAE for the extrapolating regions, b) recall, and c) precision. Average values for the 5-times random data splitting and regressions are shown. Error bars indicate standard errors. 


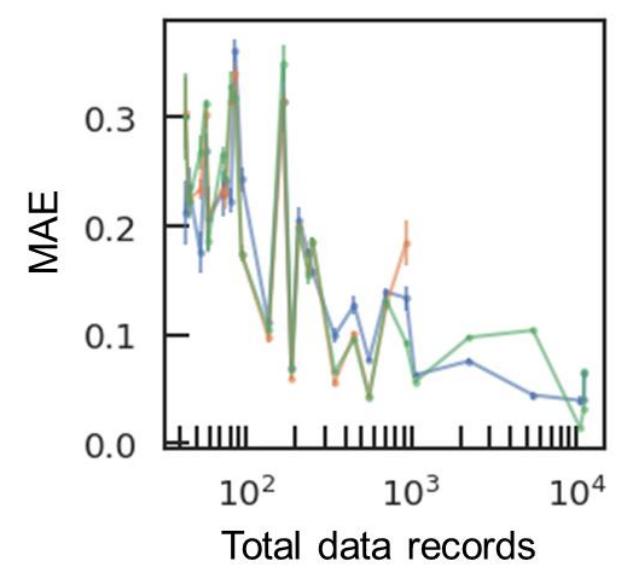

a)

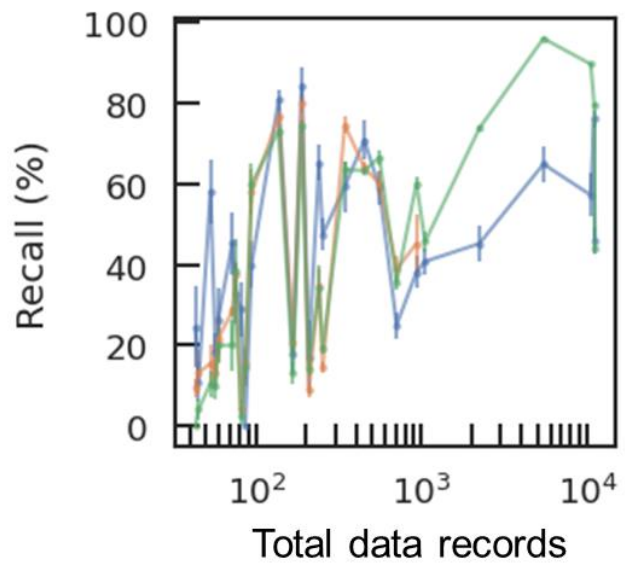

b)

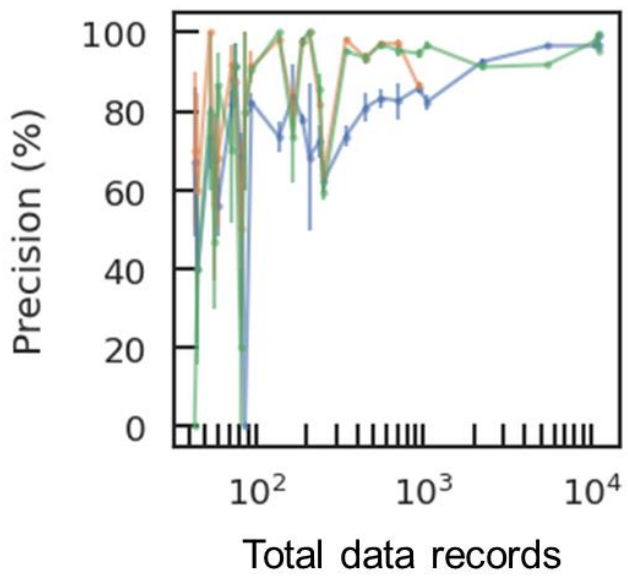

c)

Figure S14. Prediction results of Figure S13 as a function of total data records of target properties. a) MAE, b) recall, and c) precision. Error bars indicate standard errors.

\section{References}

(1) Shi, C.; Borchardt, T. B., JRgui: A Python Program of Joback and Reid Method. ACS Omega 2017, 2, $8682-8688$ 